\title{
The limit of a renewal reward process with heavy-tailed rewards is not a linear fractional stable motion
}

\author{
VLADAS PIPIRAS* and MURAD S. TAQQU** \\ Department of Mathematics, Boston University, 111 Cummington Street, Boston MA 02215, USA. \\ E-mail:*pipiras@math.bu.edu;**murad@math.bu.edu
}

Levy and Taqqu (2000) considered a renewal reward process with both inter-renewal times and rewards that have heavy tails with exponents $\alpha$ and $\beta$, respectively. When $1<\alpha<\beta<2$ and the renewal reward process is suitably normalized, the authors found that it converges to a symmetric $\beta$ stable process $\left\{Z_{\beta}(t), t \in[0,1]\right\}$ which possesses stationary increments and is self-similar. They identified the limit process through its finite-dimensional characteristic functions. We provide an integral representation for the process and show that it does not belong to the family of linear fractional stable motions.

Keywords: mixed moving average; self-similarity; stable distributions

\section{Introduction}

Renewal reward processes with heavy-tailed inter-renewal times were introduced by Mandelbrot (1969) in an economics context. Mandelbrot's model was developed by Taqqu and Levy (1986) and adapted to the telecommunications context by Taqqu et al. (1997) who considered an on-off version of the model. If the number of replications grows to infinity then, after rescaling, the limit turns out to be fractional Brownian motion. Fractional Brownian motion $B_{H}(t)$ is a Gaussian self-similar process with stationary increments. The self-similarity property means that $B_{H}(a t)$ and $a^{H} B_{H}(t)$ have the same finite-dimensional distributions for all $a>0$. The self-similarity exponent equals

$$
H=\frac{3-\alpha}{2}
$$

where $1<\alpha<2$ is the heavy-tail exponent of the inter-renewal times.

Levy and Taqqu (2000) studied what happens when the rewards are heavy-tailed as well, with exponent $\beta \in(\alpha, 2)$ (they are in the domain of attraction of a stable distribution with index $\beta$ ). They showed that, in this case, the limit process $\left\{Z_{\beta}(t), t \in[0,1]\right\}$ is a symmetric $\beta$-stable process with stationary increments which is self-similar with exponent

$$
H=\frac{\beta-\alpha+1}{\beta} .
$$


Observe that (1.2) becomes (1.1) if one sets $\beta=2$. The process $Z_{\beta}$ was only identified through its finite-dimensional distributions. But, since linear fractional stable motions (LFSMs, see (2.8) and (2.9) below) are stable counterparts of the Gaussian fractional Brownian motion, a natural conjecture is that the process $Z_{\beta}$ is an LFSM.

The purpose of this note is to show that this conjecture is false, namely that $Z_{\beta}$ is a different process. We will also provide an integral representation for $Z_{\beta}$. We will show that, while that representation bears close resemblance to the integral representation of LFSMs, $Z_{\beta}$ is, in fact, a new process, which we characterize.

\section{Integral representation and identification}

The process $Z_{\beta}$ was described in Levy and Taqqu (2000) through its finite-dimensional characteristic functions as follows. (The definition given below differs from the one in Levy and Taqqu (2000) by a multiplicative constant.) Let $0 \leqslant t_{1}, \ldots, t_{d} \leqslant 1$ be $d$ time points and $\theta_{1}, \ldots, \theta_{d}$ be arbitrary real numbers. Set $\boldsymbol{t}=\left(t_{1}, \ldots, t_{d}\right)$ and $\boldsymbol{\theta}=\left(\theta_{1}, \ldots, \theta_{d}\right)$. Then

$$
\operatorname{Eexp}\left\{\mathrm{i} \sum_{j=1}^{d} \theta_{j} Z_{\beta}\left(t_{j}\right)\right\}=\exp \{-(I(\boldsymbol{\theta}, \boldsymbol{t})+J(\boldsymbol{\theta}, \boldsymbol{t}))\},
$$

where

$$
I(\boldsymbol{\theta}, \boldsymbol{t})=\int_{0}^{\infty}\left|\sum_{j=1}^{d} \theta_{j}\left(t_{j} \wedge v\right)\right|^{\beta} v^{-\alpha} \mathrm{d} v
$$

and

$$
J(\boldsymbol{\theta}, \boldsymbol{t})=\int_{0}^{\infty} \int_{0}^{\infty}\left|\sum_{j=1}^{d} \theta_{j}\left(t_{j} \wedge v-u\right)_{+}\right|^{\beta} \alpha(v-u)_{+}^{-\alpha-1} \mathrm{~d} u \mathrm{~d} v .
$$

This work focuses on the limit process $Z_{\beta}$. We give an integral representation for $Z_{\beta}$ and provide some insight into its structure. We start by deriving its integral representation.

Proposition 2.1. The process $Z_{\beta}$ can be represented in the sense of the finite-dimensional distributions as

$$
\begin{aligned}
Z_{\beta}(t) & =\int_{-\infty}^{\infty} \int_{-\infty}^{\infty}\left((t \wedge v-u)_{+}-(0 \wedge v-u)_{+}\right) M(\mathrm{~d} u, \mathrm{~d} v) \\
& =\int_{-\infty}^{\infty} \int_{-\infty}^{\infty}\left(\int_{0}^{t} 1_{\{u<s<v\}} \mathrm{d} s\right) M(\mathrm{~d} u, \mathrm{~d} v)
\end{aligned}
$$

where $M$ is a symmetric $\beta$-stable random measure on $\mathbb{R}^{2}$ with control measure

$$
m(\mathrm{~d} u, \mathrm{~d} v)=\alpha(v-u)_{+}^{-\alpha-1} \mathrm{~d} u \mathrm{~d} v .
$$


Proof. We first show that $Z_{\beta}$ admits the representation (2.2). By Chapter 3 in Samorodnitsky and Taqqu (1994), it is enough to show that, for $\boldsymbol{t}$ and $\boldsymbol{\theta}$ as above,

$$
\int_{-\infty}^{\infty} \int_{-\infty}^{\infty}\left|\sum_{j=1}^{d} \theta_{j}\left(\left(t_{j} \wedge \boldsymbol{v}-u\right)_{+}-(0 \wedge \boldsymbol{v}-u)_{+}\right)\right|^{\beta} \alpha(\boldsymbol{v}-u)_{+}^{-\alpha-1} \mathrm{~d} u \mathrm{~d} v=I(\boldsymbol{\theta}, \boldsymbol{t})+J(\boldsymbol{\theta}, \boldsymbol{t}) .
$$

Set

$$
f_{t}(u, v)=(t \wedge v-u)_{+}-(0 \wedge v-u)_{+}
$$

and express the double integral in (2.5) as the sum of four integrals

$$
\begin{array}{r}
\left(\int_{0}^{\infty} \int_{0}^{\infty}+\int_{-\infty}^{0} \int_{0}^{\infty}+\int_{0}^{\infty} \int_{-\infty}^{0}+\int_{-\infty}^{0} \int_{-\infty}^{0}\right)\left|\sum_{j=1}^{d} \theta_{j} f_{t_{j}}(u, v)\right|^{\beta} \alpha(v-u)_{+}^{-\alpha-1} \mathrm{~d} u \mathrm{~d} v \\
=: I_{1}(\boldsymbol{\theta}, \boldsymbol{t})+I_{2}(\boldsymbol{\theta}, \boldsymbol{t})+I_{3}(\boldsymbol{\theta}, \boldsymbol{t})+I_{4}(\boldsymbol{\theta}, \boldsymbol{t}) .
\end{array}
$$

Since, for $v<0, f_{t}(u, v)=0$ the integrals $I_{2}(\boldsymbol{\theta}, \boldsymbol{t})=I_{4}(\boldsymbol{\theta}, \mathbf{t})=0$. For $v>0$ and $u>0$ $f_{t}(u, v)=(t \wedge v-u)_{+}$. Hence $I_{1}(\boldsymbol{\theta}, \mathbf{t})=J(\boldsymbol{\theta}, \mathbf{t})$. If $\boldsymbol{v}>0$ and $u<0$, then $f_{t}(u, v)=$ $t \wedge v-u-(0-u)=t \wedge v$ and, by integrating with respect to $u$, we obtain that $I_{3}(\boldsymbol{\theta}, \boldsymbol{t})=I(\boldsymbol{\theta}, \boldsymbol{t})$. This establishes relation (2.5) and, hence, representation (2.2).

To obtain (2.3) note that, for any reals $u, v$ and $t>0$,

$$
(t \wedge v-u)_{+}-(0 \wedge v-u)_{+}=\int_{0}^{t} 1_{\{u<s<v\}} \mathrm{d} s,
$$

since both sides are zero at $t=0$ and have identical derivatives for $t \neq u, t \neq v$.

Observe that the stationarity of the increments of $Z_{\beta}$ follows easily from the representation (2.3). This fact is not obvious from (2.1) (see Section 6 in Levy and Taqqu 2000). Observe also that the process $Z_{\beta}$ has continuous paths, in the sense that it admits a version which is continuous. This is because, for any $p<\beta$, the $p$ th moment $\mathrm{E}\left|Z_{\beta}(t)\right|^{p}$ is finite and, by stationarity of the increments and $H$-self-similarity of the process $Z_{\beta}$,

$$
\mathrm{E}\left|Z_{\beta}\left(t_{2}\right)-Z_{\beta}\left(t_{1}\right)\right|^{p}=\mathrm{E}\left|Z_{\beta}\left(t_{2}-t_{1}\right)\right|^{p}=\mathrm{E}\left|Z_{\beta}(1)\right|^{p}\left|t_{2}-t_{1}\right|^{H_{p}}=C\left|t_{2}-t_{1}\right|^{\mathrm{HP}} .
$$

Since

$$
H=\frac{\beta-\alpha+1}{\beta}>\frac{1}{\beta} \quad \text { or } \quad \beta>\frac{1}{H}
$$

we can choose $p<\beta$ such that $p H>1$. The existence of a continuous modification (version) of $Z_{\beta}$ then follows from (2.7) and Kolmogorov's theorem (see, for example, Theorem 12.4 in Billingsley 1968).

Can one reduce the double integral that appears in (2.3) to a single one? We would like to know, in particular, whether the process $Z_{\beta}$ is one of the LFSMs 


$$
L_{\beta}(t)=L_{\beta, H}(a, b ; t)=\int_{-\infty}^{\infty} f_{\beta, H}(a, b ; t, u) M(\mathrm{~d} u),
$$

where

$$
f_{\beta, H}(a, b ; t, u)=a\left((t-u)_{+}^{H-1 / \beta}-(-u)_{+}^{H-1 / \beta}\right)+b\left((t-u)_{-}^{H-1 / \beta}-(-u)_{-}^{H-1 / \beta}\right),
$$

$a$ and $b$ are two reals such that $|a|+|b|>0$, the self-similarity index $H$ is defined by (1.2) and $M$ is a symmetric $\beta$-stable random measure on $\mathbb{R}$ with Lebesgue control measure (see, for example, Section 7.4 in Samorodnitsky and Taqqu 1994). Observe that, since

$$
H-\frac{1}{\beta}=1-\frac{\alpha}{\beta}>0,
$$

the function $f_{\beta, H}$ in (2.9) could be expressed as

$$
f_{\beta, H}(a, b ; t, u)=a^{\prime} \int_{0}^{t}(s-u)_{+}^{H-1 / \beta-1} \mathrm{~d} s+b^{\prime} \int_{0}^{t}(s-u)_{-}^{H-1 / \beta-1} \mathrm{~d} s,
$$

where $a^{\prime}=a(H-1 / \beta)$ and $b^{\prime}=b(H-1 / \beta)$.

The following arguments may suggest a procedure for reducing the double integral in (2.3) to a single integral. As a first step, we want to interchange in (2.3) the double space integral with the time integral and study the process

$$
\int_{-\infty}^{\infty} \int_{-\infty}^{\infty} 1_{\{u<s<v\}} M(\mathrm{~d} u, \mathrm{~d} v), \quad s \geqslant 0 .
$$

Since

$$
\int_{-\infty}^{\infty} \int_{-\infty}^{\infty}\left|1_{\{u<s<v\}}\right|^{\beta}(v-u)_{+}^{-\alpha-1} \mathrm{~d} u \mathrm{~d} v=\infty,
$$

the process (2.10) is not well defined. It is easy, however, to overcome this difficulty by eliminating the high-frequency components that make the integral (2.10) diverge. Namely, for $\varepsilon>0$, we introduce the processes

$$
Z_{\beta}^{\varepsilon}(t)=\int_{-\infty}^{\infty} \int_{-\infty}^{\infty}\left(\int_{0}^{t} 1_{\{u+\varepsilon<s\}} 1_{\{s<v\}} \mathrm{d} s\right) M(\mathrm{~d} u, \mathrm{~d} v)
$$

and

$$
L_{\beta}^{\varepsilon}(t)=\int_{-\infty}^{\infty}\left(a^{\prime} \int_{0}^{t} 1_{\{u+\varepsilon<s\}}(s-u)_{+}^{H-1 / \beta-1} \mathrm{~d} s+b^{\prime} \int_{0}^{t} 1_{\{s+\varepsilon<u\}}(s-u)_{-}^{H-1 / \beta-1} \mathrm{~d} s\right) M(\mathrm{~d} u) .
$$

Observe that, when $t$ is fixed and $\varepsilon \rightarrow 0, Z_{\beta}^{\varepsilon}(t)$ and $L_{\beta}^{\varepsilon}(t)$ converge in probability to $Z_{\beta}(t)$ and $L_{\beta}(t)$, respectively. By Theorems 11.4.1 and 11.3.2 in Samorodnitsky and Taqqu (1994), the processes $Z_{\beta}^{\varepsilon}$ and $L_{\beta}^{\varepsilon}$ could be represented as

$$
Z_{\beta}^{\varepsilon}(t)=\int_{0}^{t} \tilde{Z}_{\beta}^{\varepsilon}(s) \mathrm{d} s, \quad L_{\beta}^{\varepsilon}(t)=\int_{0}^{t} \tilde{L}_{\beta}^{\varepsilon}(s) \mathrm{d} s
$$

where 


$$
\begin{gathered}
\tilde{Z}_{\beta}^{\varepsilon}(s)=\int_{-\infty}^{\infty} \int_{-\infty}^{\infty} 1_{\{u+\varepsilon<s\}} 1_{\{s<v\}} M(\mathrm{~d} u, \mathrm{~d} v), \\
\tilde{L}_{\beta}^{\varepsilon}(s)=\int_{-\infty}^{\infty}\left(a^{\prime} 1_{\{u+\varepsilon<s\}}(s-u)_{+}^{H-1 / \beta-1}+b^{\prime} 1_{\{s+\varepsilon<u\}}(s-u)_{-}^{H-1 / \beta-1}\right) M(\mathrm{~d} u) .
\end{gathered}
$$

We will focus on the stationary processes $\tilde{Z}_{\beta}^{\varepsilon}$ and $\tilde{L}_{\beta}^{\varepsilon}$ instead of considering the processes $Z_{\beta}^{\varepsilon}$ and $L_{\beta}^{\varepsilon}$ which have stationary increments.

The second step of the argument involves performing the integration over $v$ in (2.12). If we fix $s$ and set

$$
\tilde{M}(\mathrm{~d} u)=\int_{-\infty}^{\infty} 1_{\{u<s<v\}} M(\mathrm{~d} u, \mathrm{~d} v),
$$

then $\tilde{M}$ is a symmetric $\beta$-stable random measure on $\mathbb{R}$ with control measure

$$
\begin{aligned}
\tilde{m}(\mathrm{~d} u) & =\int_{-\infty}^{\infty} 1_{\{u<s<v\}} \alpha(v-u)_{+}^{-\alpha-1} \mathrm{~d} u \mathrm{~d} v \\
& =(s-u)_{+}^{-\alpha} \mathrm{d} u=\left((s-u)_{+}^{H-1 / \beta-1}\right)^{\beta} \mathrm{d} u .
\end{aligned}
$$

In view of (2.14), we can set

$$
\left.\tilde{Z}_{\beta}^{\varepsilon}(s) \stackrel{d}{=} \int_{-\infty}^{\infty} 1_{\{u+\varepsilon<s\}}(s-u)_{+}^{H-1 / \beta-1} M(\mathrm{~d} u) \quad \text { (fixed } s\right),
$$

where $M$ is stable with Lebesgue control measure. Comparing (2.15) with (2.13), we would expect that the process $\tilde{Z}_{\beta}^{\varepsilon}$ has the same finite-dimensional distributions as the process $\tilde{L}_{\beta}^{\varepsilon}$, when $a^{\prime}=1$ and $b^{\prime}=0$, and therefore that the process (2.3) reduces to an LFSM. Since $\tilde{M}$ depends on $s$, this is true for the marginal distributions (fixed time point $s$ ) but is not obvious when one considers the distribution of the vector

$$
\left(\tilde{Z}_{\beta}^{\varepsilon}\left(s_{1}\right), \ldots, \tilde{Z}_{\beta}^{\varepsilon}\left(s_{d}\right)\right), \quad d \geqslant 2 .
$$

In fact, the following proposition shows that the process $Z_{\beta}$ is not an LFSM.

Proposition 2.2. The process $Z_{\beta}$ in (2.2) is not one of the linear fractional stable motions $L_{\beta}$ in (2.8).

Proof. We will proceed by contradiction. Assume that there exist two reals $a$ and $b$ such that

$$
\left\{Z_{\beta}(t), t \in[0,1]\right\} \stackrel{d}{=}\left\{L_{\beta, H}(a, b ; t), t \in[0,1]\right\},
$$

where the equality holds in the sense of the finite-dimensional distributions. Since both processes are self-similar with exponent $H$ and have stationary increments, we may assume that $t \in \mathbb{R}$ in (2.16). By Theorem 3.1 in Rosiński (1994), there exist two Borel functions $\phi=\left(\phi_{1}, \phi_{2}\right): \mathbb{R} \rightarrow \mathbb{R}^{2}$ and $h: \mathbb{R} \rightarrow \mathbb{R}$ such that 


$$
\begin{aligned}
a\left((t-u)_{+}^{H-1 / \beta}-(-u)_{+}^{H-1 / \beta}\right) & +b\left((t-u)_{-}^{H-1 / \beta}-(-u)_{-}^{H-1 / \beta}\right) \\
& =h(u)\left(\left(t \wedge \phi_{1}(u)-\phi_{2}(u)\right)_{+}-\left(0 \wedge \phi_{1}(u)-\phi_{2}(u)\right)_{+}\right)
\end{aligned}
$$

$\mathrm{d} t \mathrm{~d} u$ a.e. Fix $u=u_{0}$ for which this equation holds $\mathrm{d} t$ a.e. By taking $t>0$ large enough, we necessarily have $a=0$. Equation (2.17) is then not possible as $t$ tends to $-\infty$ because its one side is asymptotic to $b|t|^{H-1 / \beta}$, the other to $h\left(u_{0}\right) t$ and $H-1 / \beta=1-\alpha / \beta \neq 1$. Hence assumption (2.16) is wrong.

How then to characterize $Z_{\beta}$ ? Since its derivative $Z_{\beta}^{\prime}$ is not defined, we will instead use the stationary process $\tilde{Z}_{\beta}^{\varepsilon}$ introduced earlier. Were $Z_{\beta}$ a linear fractional stable motion, then $\tilde{Z}_{\beta}^{\varepsilon}$ would be a moving average. As we will show, $\tilde{Z}_{\beta}^{\varepsilon}$ is a 'mixed moving average'.

Definition 2.1. A symmetric $\beta$-stable (S $\beta \mathrm{S})$ stationary process $\left\{X_{s}\right\}_{s \in \mathbb{R}}$ is a mixed moving average if there is a Borel space E equipped with a $\sigma$-finite measure $v$ on $E$ and a function $g \in L^{\beta}(\mathbb{R} \times E$, Leb $\otimes v)$ such that

$$
\left\{X_{s}\right\}_{s \in \mathbb{R}} \stackrel{d}{=}\left\{\int_{\mathbb{R} \times E} g(s-u, x) \tilde{M}(\mathrm{~d} u, \mathrm{~d} x)\right\}_{s \in \mathbb{R}},
$$

in the sense of the finite-dimensional distributions. Here $\tilde{M}$ is a $\mathrm{S} \beta \mathrm{S}$ random measure on $\mathbb{R} \times E$ with the control measure Leb $\otimes v$. (A Borel space is a measurable space which is measurably isomorphic to a Borel subset of the real line, that is, there exists a one-to-one and onto map $\psi$ between the two sets such that both $\psi$ and $\psi^{-1}$ are measurable.)

The class of mixed moving averages was introduced by Surgailis et al. (1993) as an important extension of the usual moving averages. It preserves ergodicity and is closed under linear combinations. This class also appears in the decomposition of a stationary symmetric stable process established by Rosiński (1995).

Proposition 2.3. The $\mathrm{S} \beta \mathrm{S}$ stationary process $\tilde{Z}_{\beta}^{\varepsilon}$ corresponding to the stationary increment process $Z_{\beta}$ is a mixed moving average.

Proof. Rewrite (2.12) as

$$
\left\{\tilde{Z}_{\beta}^{\varepsilon}(s)\right\}_{s \in \mathbb{R}}=\left\{\int_{\mathbb{R} \times \mathbb{R}} 1_{\{\varepsilon<s-u<v-u\}} M(\mathrm{~d} u, \mathrm{~d} v)\right\}_{s \in \mathbb{R}}
$$

and make the change in variables $u \rightarrow u$ and $v-u \rightarrow x$ to obtain a mixed moving average representation

$$
\begin{aligned}
\left\{\tilde{Z}_{\beta}^{\varepsilon}(s)\right\}_{s \in \mathbb{R}} & =\left\{\int_{\mathbb{R} \times \mathbb{R}} 1_{\{\varepsilon<s-u<x\}} \tilde{M}(\mathrm{~d} u, \mathrm{~d} x)\right\}_{s \in \mathbb{R}} \\
& =\left\{\int_{\mathbb{R} \times \mathbb{R}} g(s-u, x) \tilde{M}(\mathrm{~d} u, \mathrm{~d} x)\right\}_{s \in \mathbb{R}},
\end{aligned}
$$


where $g(u, x)=1_{\{\varepsilon<u<x\}}$ and $\tilde{M}$ is a $\mathrm{S} \beta \mathrm{S}$ random measure on $\mathbb{R} \times \mathbb{R}$ with control measure $\tilde{M}(\mathrm{~d} u, \mathrm{~d} x)=\alpha x_{+}^{-\alpha-1} \mathrm{~d} u \mathrm{~d} x$.

Observe that $\tilde{Z}_{\beta}^{\varepsilon}$ can then be rewritten as

$$
\tilde{Z}_{\beta}^{\varepsilon}(s)=\alpha^{1 / \beta} \int_{-\infty}^{\infty} \int_{-\infty}^{\infty} 1_{\{\varepsilon<s-u<x\}} x_{+}^{H-2 / \beta-1} M(\mathrm{~d} u, \mathrm{~d} x),
$$

where $M$ is a $\mathrm{S} \beta \mathrm{S}$ random measure on $\mathbb{R} \times \mathbb{R}$ with Lebesgue control measure $m(\mathrm{~d} u, \mathrm{~d} x)=\mathrm{d} u \mathrm{~d} x$. While representation (2.15) holds only for a fixed $s$, (2.19) holds in the sense of the finite-dimensional distributions. Consequently, for the process $Z_{\beta}$, which has stationary increments, we obtain:

Corollary 2.1. The process $Z_{\beta}$ can be expressed as

$$
Z_{\beta}(t)=(\mathrm{P}) \lim _{\varepsilon \rightarrow 0} \int_{0}^{t} \tilde{Z}_{\beta}^{\varepsilon}(s) \mathrm{d} s,
$$

where $\tilde{Z}_{\beta}^{\varepsilon}$ has the mixed moving average representation (2.18) or (2.19) and where (P)lim denotes the limit in probability.

Remark. The covariance is not defined for $\beta$-stable processes with $\beta<2$. One uses instead other measures of dependence such as the covariation and the codifference (see Samorodnitsky and Taqqu 1994). The asymptotic behaviour of the covariation and the codifference for the stationary increment process $Z_{\beta}(t+1)-Z_{\beta}(t)$ is given in Levy and Taqqu (1999).

\section{Acknowledgements}

This research was partially supported by NSF grants DMS-9404093 and ANI-9805623 at Boston University.

\section{References}

Billingsley, P. (1968) Convergence of Probability Measures. New York: Wiley.

Levy, J.B. and Taqqu, M.S. (1999) Asymptotic rates for the covariation and the codifference of a renewal-reward process with infinite variance. Preprint.

Levy, J.B. and Taqqu, M.S. (2000) Renewal reward processes with heavy-tailed inter-renewal times and heavy-tailed rewards. Bernoulli, 6, 23-44.

Mandelbrot, B.B. (1969) Long-run linearity, locally Gaussian processes, $H$-spectra and infinite variances. Internat. Econom. Rev., 10, 82-113.

Rosiński, J. (1994) On uniqueness of the spectral representation of stable processes. J. Theoret. Probab., 7(3), 615-634.

Rosiński, J. (1995) On the structure of stationary stable processes. Ann. Probab., 23, 1163-1187.

Samorodnitsky, G. and Taqqu M.S. (1994) Stable Non-Gaussian Processes: Stochastic Models with 
Infinite Variance. London: Chapman \& Hall.

Surgailis, D., Rosiński, J., Mandrekar, V. and Cambanis, S. (1993) Stable generalized moving averages. Probab. Theory Related Fields, 97, 543-558.

Taqqu, M.S. and Levy, J. (1986) Using renewal processes to generate long-range dependence and high variability. In E. Eberlein and M.S. Taqqu (eds), Dependence in Probability and Statistics, pp. 73-89. Boston: Birkhäuser.

Taqqu, M.S., Willinger, W. and Sherman, R. (1997) Proof of a fundamental result in self-similar traffic modeling. Comput. Commun. Rev., 27(2), 5-23.

Received January 1999 